PALAVRA ABERTA / FREE SPEECH 


\title{
EL SISTEMA UNIVERSITARIO EN EL CHILE CONTEMPORÁNEO
}

\author{
Felipe Andres Zurita Garrido* \\ Universidad Academia de Humanismo Cristiano (UAHC)
}

\section{LA PROVOCACIÓN}

A propósito de la crisis financiera que atraviesa la Universidad de Sao Paulo (USP) con mucha sorpresa encontré en algunos medios de comunicación masivos de Brasil una referencia levemente positiva al modelo de financiamiento universitario de Chile, que se basa en el cobro de aranceles a sus estudiantes. Dicha referencia se presentaba como posible ejemplo a seguir con miras a que dicha institución pudiese obtener recursos. Fue tanto el entusiasmo, que incluso el periódico Folba de Sao Paulo (02/06/2014) llegó a realizar el cálculo de cuántos estudiantes de la USP podrían pagar una mensualidad y cuánto dinero significaría esto anualmente. ${ }^{1}$ El entusiasmo creció aún más y, a propósito de que la misma USP fue superada por la Pontificia Universidad Católica de Chile (PUC) como la mejor Institución de Educación Superior Latinoamericana según el Ranking $\mathrm{QS}^{2}$, en la Revista Veja (13/07/2014) se publicó una entrevista al Rector de la PUC, Ignacio Sánchez, donde, además de explicar cómo la institución que dirige logró alcanzar dicha posición, aprovechó de realizar una defensa abierta del modelo de financiamiento basado en el cobro de mensualidades a los estudiantes en Chile. Una coincidencia.

Frente a esta situación y con el ánimo de poder entregar al público de Brasil una mirada diferente sobre el sistema universitario de Chile, he decidido escribir este texto.

\section{1: LA DESTRUCCIÓN/INVENCIÓN DE LA UNIVERSIDAD EN CHILE}

La historia de la Universidad en Chile es larga. Ya en la Época Colonial, cuando el actual territorio de Chile era una posesión del Imperio Español, es posible identificar el surgimiento de las primeras instituciones de este tipo:

\footnotetext{
http://dx.doi.org/10.1590/0102-4698138059

* Magíster en Educación por la Universidad Academia de Humanismo Cristiano (UAHC). Profesor de la Carrera de Pedagogía en Historia y Ciencias Sociales, Facultad de Pedagogía, Universidad Academia de Humanismo Cristiano, Chile. Estudiante del Programa de Doctorado Latinoamericano en Educación: Políticas Públicas y Profesión Docente e integrante del Grupo de Estudos e Pesquisas em História da Educação (GEPHE), Facultad de Educación, Universidad Federal de Minas Gerais, Brasil. E-mail: felipe_zuritag@yahoo.es
} 
- Universidad Pontificia de Santo Tomás de Aquino (16221747) perteneciente a la Orden Dominicana. Otorgaba Títulos Superiores en Teología.

- Convictorio San Francisco Javier (1623-1767) perteneciente a la Orden Jesuita. Se abocaba fundamentalmente a la formación de religiosos. ${ }^{3}$

- Real Universidad de San Felipe (1747-1843). Universidad perteneciente a la Corona Española. Otorgaba Títulos de Teología, Medicina, Derecho, Filosofía, Matemáticas. ${ }^{4}$

Con posterioridad, ya en la Época Republicana, el naciente Estado de Chile y grupos de la sociedad civil comenzaron poco a poco a fundar Universidades con miras a ponerlas al servicio de la construcción del país, la formación de profesionales, como así también del desarrollo económico, cultural, científico y social.

TABLA 1

Universidades Chilenas Fundadas entre 1810-1981

\begin{tabular}{|l|c|c|}
\hline \multicolumn{1}{|c|}{ Nombre } & Fundación & Propiedad \\
\hline Universidad de Chile & 1842 & Estatal \\
\hline Pontificia Universidad Católica de Chile & 1888 & Privada \\
\hline Universidad de Concepción & 1919 & Privada \\
\hline Pontificia Universidad Católica de Valparaíso & 1925 & Privada \\
\hline Universidad Técnica Federico Santa María & 1931 & Privada \\
\hline Universidad Técnica del Estad0 ${ }^{5}$ & 1947 & Estatal \\
\hline Universidad Austral de Chile & 1954 & Privada \\
\hline Universidad del Norte & & Privada \\
\hline
\end{tabular}

Fuente: PIIE (1984)

Hacia finales de la década de 1960 el sistema universitario chileno se vio removido por un interesante proceso de Reforma Universitaria que bregaba por la democratización interna de la dirección de las instituciones, por alcanzar una vinculación mayor con la comunidad nacional y por abrirse a aquellos sectores sociales y culturales históricamente excluidos. De esta manera, se buscaba poner la Universidad al servicio del desarrollo nacional desde una perspectiva democrática y pluralista. ${ }^{7}$ Este proceso de Reforma Universitaria fue 
violentamente paralizado con el Golpe de Estado del 11 de septiembre de 1973 que derrocó al gobierno democráticamente electo de Salvador Allende y que puso en el poder a una Junta Militar liderada posteriormente por Augusto Pinochet Ugarte, dando paso así al desarrollo de una Dictadura Militar sangrienta de 17 años (1973-1990).

¿Qué hizo la Dictadura Militar con las Universidades? Una de las primeras medidas tomadas fue la publicación del Decreto Ley $\mathrm{N}^{\circ} 50(02 / 10 / 1973)$ que establecía claramente la intervención del régimen en las Universidades a través de la creación de la figura de los Rectores-Delegados, que de allí hasta el término de la Dictadura Militar dirigirían a estas instituciones. Por otra parte y en una línea de acción menos pública, la Dictadura Militar persiguió a trabajadores, académicos y estudiantes de izquierda, y/o comprometidos con perspectivas transformacionales de la sociedad chilena, pertenecientes a diversas Universidades, expulsándolos y despidiéndolos de las mismas, y también en muchos casos encarcelándolos torturándolos, asesinándolos y desapareciéndolos. ${ }^{8}$ De esta manera, los campus de estas instituciones fueron mudos testigos de una sangrienta cacería ideológica, donde quedó muy claro que se excluiría abiertamente a la actividad política opositora al régimen y a la política misma como expresión posible dentro del mundo universitario.

Posteriormente, aún bajo la Dictadura Militar en el año 1981, viene la gran transformación al sistema universitario chileno a través de la publicación del Decreto Con Fuerza de Ley $\mathrm{N}^{\circ} 1$ denominada Ley General de Universidades. Lo que allí se definió en términos generales es lo siguiente:

- Limitación de la autonomía universitaria (Articulos 3, 4, 5, 6, 7 e 8).

- Exclusión de la política partidista como expresión posible dentro de las Universidades. (Artículo 6).

- Establecimiento de un conjunto de Títulos Profesionales que solo podrán desarrollarse dentro de las Universidades yaque necesitarían de la obtención del Grado Académico de Licenciado. (Artículos 11, 12 y 13).

- Autorización para crear Universidades Privadas, en tanto "personas jurídicas de derecho privado sin fines de lucro". (Artículo 15).

- Prohibición de la participación de los funcionarios administrativos y estudiantes en la definición de la gestión y definición de la dirección de estas nuevas Universidades. (Artículo $24^{\circ}$ ).

Además de lo anteriormente señalado, se dio paso al desmembramiento de la Universidad de Chile (UCH) y de la 
Universidad Técnica del Estado (UTE), dando paso a la creación de nuevas Universidades Estatales a partir de las sedes regionales que estas instituciones tenían. En esta línea, el escenario de las Universidades Estatales se diversifica a partir de la eliminación forzosa de la presencia de carácter nacional que estas dos instituciones tenían a lo largo del país. Dichas sedes regionales fueron transformadas directamente, o fusionadas con otras sedes, para dar paso a las nuevas Universidades Estatales. El escenario pasó a ser de la siguiente manera:

\section{TABLA 2}

Desmembramiento Universidad de Chile y Universidad Técnica del Estado

\begin{tabular}{|c|c|c|}
\hline $\begin{array}{c}\text { Universidad } \\
\text { Estatal }\end{array}$ & Sede Regional & Nueva Universidad Estatal \\
\hline \multirow{13}{*}{$\begin{array}{l}\text { Universidad de } \\
\text { Chile }\end{array}$} & Sede Arica & Universidad de Tarapacá \\
\hline & Sede Iquique & Universidad Arturo Prat \\
\hline & Sede Antofagasta & Universidad de Antofagasta \\
\hline & Sede La Serena & Universidad de La Serena \\
\hline & Sedea Valparaíso & Universidad de Valparaíso \\
\hline & Instituto Pedagógico de Valparaíso & Universidad de Playa Ancha de Ciencias de la Educación ${ }^{10}$ \\
\hline & Sede Santiago & Universidad de Chile \\
\hline & Instituto Profesional de Santiago & Universidad Tecnológica Metropolitana \\
\hline & Instituto Pedagógico de Santiago & Universidad Metropolitana de Ciencias de la Educación ${ }^{11}$ \\
\hline & Sede Talca & Universidad de Talca \\
\hline & Sede Chillán & Universidad del Bío-Bío \\
\hline & Sede Temuco & Universidad de La Frontera \\
\hline & Sede Osorno & Universidad de Los Lagos \\
\hline \multirow{9}{*}{$\begin{array}{l}\text { Universidad } \\
\text { Técnica del } \\
\text { Estado }\end{array}$} & Sede Antofagasta & Universidad de Antofagasta \\
\hline & Sede Copiapó & Universidad de Atacama \\
\hline & Sede La Serena & Universidad de La Serena \\
\hline & Sede Santiago & Universidad de Santiago de Chile \\
\hline & Sede Talca & Universidad de Talca \\
\hline & Sede Concepción & Universidad del Bío-Bío \\
\hline & Sede Temuco & Universidad de La Frontera \\
\hline & Sede Osorno & Universidad de Los Lagos \\
\hline & Sede Punta Arenas & Universidad de Magallanes \\
\hline
\end{tabular}

Fuente: PIIE (1984) 
En base a estas transformaciones se pasa del escenario de 1981 compuesto por 8 Universidades ( 2 estatales y 6 privadas), a un escenario radicalmente diferente, donde en la actualidad existen 60 Universidades diferenciadas según 2 grandes tipologías:

1. Universidades pertenecientes al Consejo de Rectores de las Universidades de Chilenas (CRUCH):

a) Universidades Estatales: 16 Universidades que se originan del desmembramiento de la Universidad de Chile y de la Universidad Técnica del Estado.

b) Universidades Privadas Tradicionales: 9 Universidades que corresponden a las 6 Universidades Privadas creadas antes de 1981 y a otras que nacieron a partir de estas

2. Universidades Privadas: 35 Universidades fundadas posteriormente a 1981. Son de una alta variedad, algunas de propiedad de grupos académicos, empresariales, políticos y religiosos diferenciados, y además, otras pertenecientes a transnacionales dedicadas al negocio educacional. ${ }^{12}$

El aumento de la cantidad de Universidades en Chile se realizó en base al convencimiento de la autoridad con respecto a los supuestos beneficios que tendría una presencia mayor de la iniciativa privada en la otorgación de posibilidades educativas a la población. Claramente lo que primó fue la aplicación de una ortodoxia neoliberal basada en la idea de la necesidad de un "mercado universitario" organizado en base a la competencia entre las diferentes Universidades por recursos y estudiantes, lo que permitiría la constante mejora de los niveles de calidad de dichas instituciones. Para que dicha competencia fuese verdadera se desenvolvió un paulatino, pero claro a la vez, proceso de desfinanciamiento de las Universidades por parte del Estado, en base al argumento de que aquí en adelante quienes asistan a dicha institución tendrán que pagar matrículas y aranceles mensuales por ello. De esta manera, lo que en Chile ocurrió fue un evidente paso forzado de una lógica de Universidad en creciente democratización, gratuita y pública a una lógica de Universidad elitista, pagada y privada. Todo por supuesto en base a la ausencia de evidencia científica y de cualquier debate democrático posible, en otras palabras, sin ninguna legitimidad más allá de la fuerza.

En concordancia con el aumento de Universidades se vivió también un aumento de la matrícula: 
TABLA 3

Matrícula Universidades Chilenas 1983-2013

\begin{tabular}{|c|c|c|c|}
\hline Año & $\begin{array}{c}\text { Matrícula Universidades } \\
\text { CRUCH }^{13}\end{array}$ & $\begin{array}{c}\text { Matrícula Universidades } \\
\text { Privadas }\end{array}$ & $\begin{array}{l}\text { Total de la Matrícula } \\
\text { Universitaria }\end{array}$ \\
\hline 1983 & 115.865 & 2.708 & 118.573 \\
\hline 1984 & 118.284 & 3.686 & 121.970 \\
\hline 1985 & 121.612 & 4.951 & 126.563 \\
\hline 1986 & 122.127 & 5.235 & 127.362 \\
\hline 1987 & 119.447 & 7.652 & 127.099 \\
\hline 1988 & 116.283 & 9.246 & 125.529 \\
\hline 1989 & 105.590 & 13.773 & 119.363 \\
\hline 1990 & 112.193 & 19.509 & 131.702 \\
\hline 1991 & 117.892 & 28.828 & 146.720 \\
\hline 1992 & 127.602 & 40.706 & 168.308 \\
\hline 1993 & 144.346 & 50.270 & 194.616 \\
\hline 1994 & 151.570 & 59.994 & 211.564 \\
\hline 1995 & 161.850 & 69.377 & 231.227 \\
\hline 1996 & 174.958 & 78.565 & 253.523 \\
\hline 1997 & 184.089 & 85.506 & 269.595 \\
\hline 1998 & 199.973 & 87.697 & 287.670 \\
\hline 1999 & 206.576 & 92.821 & 299.397 \\
\hline 2000 & 215.284 & 103.805 & 319.089 \\
\hline 2001 & 227.392 & 111.963 & 339.355 \\
\hline 2002 & 243.610 & 125.917 & 369.527 \\
\hline 2003 & 246.750 & 156.620 & 403.370 \\
\hline 2004 & 246.577 & 170.410 & 416.987 \\
\hline 2005 & 247.969 & 193.177 & 441.146 \\
\hline 2006 & 262.151 & 215.924 & 478.075 \\
\hline 2007 & 285.984 & 233.573 & 519.557 \\
\hline 2008 & 295.158 & 251.050 & 546.208 \\
\hline 2009 & 303.127 & 273.473 & 576.600 \\
\hline 2010 & 310.890 & 323.843 & 634.843 \\
\hline 2011 & 309.333 & 352.529 & 661.862 \\
\hline 2012 & 311.775 & 374.202 & 685.977 \\
\hline 2013 & 326.040 & 381.894 & 707.934 \\
\hline
\end{tabular}

Fuente: MINEDUC (2013) 
A partir del cuadro anterior es posible señalar que existió un incremento generalizado de la matrícula en las Universidades chilenas en los últimos 30 años, no obstante, existen algunos matices que señalar. Primero, es notorio el estancamiento en la matrícula de las Universidades del CRUCH durante la Dictadura Militar, parece como que intencionalmente se hubiese desincentivado el potenciamiento de estas en favor de las nacientes Universidades Privadas. Segundo, es impactante el avance avasallador de la matrícula de las Universidades Privadas, que en base a un ritmo de crecimiento anual gigantesco logró por fin en el año 2010 superar la matrícula de las Universidades del CRUCH. Tercero, es interesante que el aumento más ostensible de las Universidades Privadas se diera una vez terminada la Dictadura Militar, o sea, que en pleno periodo de los Gobiernos Democráticos o de Post-Dictadura (1990 en adelante) el modelo de Universidad Privada encuentra un espacio aún más fértil para crecer.

Este aumento exponencial de la matrícula universitaria, con fuerte expansión del sector privado, ya no fue financiado en su parte mayoritaria por el Estado como en el pasado, sino que se pasó a una modalidad de financiamiento sustentado en dos grandes focos de recursos:

- Aportes Fiscales: ${ }^{14}$

- Aporte Fiscal Directo (AFD): corresponde a remesas anuales entregadas por el Estado y repartidas entre las Universidades pertenecientes al CRUCH y calculado en base a criterios históricos.

- Aporte Fiscal Indirecto (AFI): corresponde a un aporte que entrega al Estado a cualquier Universidad ${ }^{15}$ que recibe a los 27.500 mejores puntajes en la Prueba de Selección Universitaria (PSU).

- Aportes Privados:

- Donaciones.

- Venta de cursos de extensión.

- Prestaciones de servicios a empresas.

- Aranceles cobrados a los estudiantes.

Los aranceles o mensualidades pagadas por los estudiantes corresponden a la proporción mayor de ingresos monetarios recibidos por las Universidades, tanto del CRUCH como Privadas. Según datos del Ministerio de Educación (2012) los aranceles cobrados a los estudiantes representaron los siguientes porcentajes del total del financiamiento anual de las Universidades chilenas: 
- Universidades del CRUCH: $42,42 \%$.

- Universidades Privadas: 79,02\%

- Universidades en total: $57,32 \%$

De esta forma, el sistema universitario chileno se sostiene en la actualidad claramente en base a un fuerte impacto en la renta de las familias chilenas. Además, según datos de la OCDE para el año $2013^{16}$ el promedio de costo pagado por los estudiantes en las Universidades chilenas es uno de los más caros a nivel mundial, bordeando los U\$6.000 anuales, superando así por mucho al costo de las Universidades más prestigiosas y reconocidas a nivel mundial. Para poder financiar ese gasto, y también para aumentarlo en cierta medida, se creó en el año 2005 el Crédito con Aval del Estado CAE (Ley 20.027) que se constituyó en una modalidad de financiamiento directo a los estudiantes para que pudiesen financiar sus estudios. ${ }^{17}$ Esta modalidad permitió el acceso masivo a la Universidad a miles de estudiantes que no tenían las condiciones económicas para pagar las altas mensualidades. No obstante, por otra parte, generó un problema de endeudamiento radicalmente costoso para los estudiantes, puesto que inicialmente el crédito se entregaba con tasas de interés anuales por sobre el promedio de mercado (cercana al 5,8\%) generando así un rápido aumento del valor total a pagar a futuro. Además de lo anterior, la fácil y masiva obtención de créditos por parte de los estudiantes se constituyó en un incentivo para que muchas Universidades aumentaran a toda costa tanto la cantidad de estudiantes matriculados, como así también el valor anual de las mensualidades. Cuando comenzaron a egresar los primeros estudiantes que financiaron sus estudios con este crédito, se hizo evidente que el mismo había sido pensado y utilizado más como una oportunidad de negocio fácil y seguro para las instituciones educacionales y financieras que se veían beneficiadas por el mismo, y menos como una herramienta justa y adecuada de facilitación de acceso a financiamiento para cursar estudios universitarios. Al día de hoy hay cerca de 350.000 estudiantes en Chile profundamente endeudados con este crédito.

Otra de las características centrales del actual sistema universitario es su modalidad de evaluación de la calidad. La ya mencionada expansión de las Universidades y de las matrículas fue acompañada de una tenue fiscalización, en tanto que lo que predominó desde la perspectiva de la autoridad fue la confianza excesiva en la honestidad y buena fe que aseguraría la iniciativa privada. Dicha confianza sólo es posible de entender en un contexto ideológicamente sustentado en la supuesta superioridad e 
incorruptibilidad de la iniciativa privada por sobre la iniciativa pública/ estatal. Es sólo cuando el sistema universitario se veía casi inmanejable donde se optó por establecer un mecanismo de evaluación del mismo. Fue así como fueron creadas, primero, la Comisión Nacional de Acreditación de Pregrado (Decreto Supremo No 51 06/04/1999) y después la Comisión Nacional de Acreditación (Ley N 20.129 17/11/2006). La idea era regular el sistema universitario con miras a determinar la calidad de los servicios educacionales vendidos a los estudiantes. De esta forma, si bien el proceso de Acreditación es voluntario, ${ }^{18}$ se estableció un nexo entre el Crédito con Aval del Estado (CAE) y la obligatoriedad de someterse al proceso de Acreditación. En otras palabras, para poder matricular estudiantes con el CAE era necesario que la Universidad estuviese acreditada. Frente a esta situación, las Universidades se vieron obligadas a someterse al proceso de revisión en tanto necesitaban los recursos. ${ }^{19}$

Algunos aspectos del proceso de Acreditación han llamado la atención. Primero es muy llamativo que la Comisión Nacional de Acreditación sea integrada por un conjunto de Comisionados, dentro de los cuales están presentes, con derecho a voz y voto, representantes de las Universidades del CRUCH (3 Comisionados), Universidades Privadas (2 Comisionados), Institutos Profesionales (1 Comisionado) y Centros de Formación Técnica (1 Comisionado). De esta manera, de un total de 15 Comisionados, 7 corresponden a personas vinculadas a las mismas instituciones que se deben poner en posición de evaluado. De esta forma, los alcances de esta institucionalidad quedan, de una u otra forma, al alcance de la mano de las mismas instituciones a ser evaluadas.

Segundo, en el año 2012 fue conocido por la opinión pública que el entonces Presidente de la Comisión Nacional de Acreditación durante el año 2010, Luis Eugenio Díaz, habría sido contratado para realizar asesorías a Universidades que después fueron acreditadas estando él dirigiendo todo el proceso. Esta situación dio paso a un proceso de investigación judicial que desembocó en la prisión de Luis Eugenio Díaz acusado por corrupción y además con la credibilidad del sistema de Acreditación y de las Universidades involucradas por los suelos.

Tercero, existen 5 grandes áreas ${ }^{20}$ a evaluar de una Universidad según la Comisión Nacional de Acreditación:

- Gestión Institucional.

- Docencia de Pregrado.

- Docencia de Postgrado.

- Investigación.

- Vinculación con el Medio. 
Lo interesante es que si una Universidad busca evaluar su calidad en tanto tal, solo está obligada a ser revisada en las dos primeras áreas, siendo las tres restantes voluntarias. De esta manera, la misma institucionalidad que revisa la calidad de las Universidades chilena posibilita la omisión legal de dimensiones fundamentales del funcionamiento y misión de la Universidad, en tanto instancia productora de conocimiento y al servicio de una comunidad más amplia que el cuerpo de trabajadores, académicos y estudiantes que se encuentran dentro de sus muros. En otras palabras, es posible sostener que se propone aquí una reducción legal de la misma idea de Universidad.

Los resultados del proceso de Acreditación de las Universidades chilenas son interesantes de observar, puesto que muestran la diversidad de instituciones y desempeños existentes. Por un lado, solamente 2 instituciones han sido capaces de confirmar su excelencia en todas las áreas establecidas por el sistema, se trata de la Universidad de Chile (UCH) y de la Pontificia Universidad Católica de Chile (PUC). Mientras que en el otro extremo, hoy día hay 15 Universidades que no se encuentran Acreditadas. ${ }^{21}$ Entre estos dos extremos existe una diversidad de instituciones que ameritaría una revisión exhaustiva en otra instancia. No obstante, sí es necesario señalar que un grupo importante de estas han sido objeto de investigaciones por parte de la autoridad, en tanto existen fuertes sospechas y evidencias concretas de que estarían infringiendo la ley en tanto habrían lucrado con los recursos percibidos, generando condiciones logísticas y de organización interna tanto para lucrar, como así también para ocultar dicha actividad. ${ }^{22}$

El año 2011 fue el momento en que los estudiantes de diferentes niveles educacionales de Chile salieron a las calles, apoyados por la inmensa mayoría de la población del país, para cuestionar este modelo de educación organizado en la obtención de lucro por parte de proveedores privados, que ponen sus intereses corporativos por sobre cualquier miramiento en torno de la calidad educacional y la dignidad humana. Uno de los focos centrales de las reivindicaciones de los estudiantes fue justamente la figura de pago de aranceles para cursar estudios en las Universidades, en tanto mecanismo que afecta profundamente, y a largo plazo, la renta de las familias chilenas y además reduce el funcionamiento de estas instituciones a una maquinaria productora de dinero. La demanda de los estudiantes es justamente instaurar un sistema de financiamiento estatal, que permita la gratuidad de los estudios universitarios para toda la población en tanto derecho social, independiente de la 
renta familiar de cada persona. Hasta ahora eso aún no ha sido alcanzado en Chile, a pesar del apoyo mayoritario de la población a dicha demanda. Ciertamente esto se explica en que la clase política chilena se encuentra íntimamente ligada y comprometida, en tanto propietarios muchas veces, con los intereses económicos y políticos que sustentan este sistema. Esta situación claramente implicará nuevas y prolongadas instancias de presión popular con miras a obligar a las autoridades políticas del país a asumir dicha aspiración. En el caso de Brasil las Universidades Públicas aún son gratuitas tanto a nivel de Pregrado como de Postgrado. Esa es una ganancia de fuerte relevancia para la ciudadanía brasilera que tiene que ser protegida y extendida con miras a que cada vez más aquellos grupos históricamente excluidos de la Universidad tengan un acceso mayor, en base a criterios de justicia, calidad y dignidad. Para seguir ese camino, el ejemplo chileno claramente no amerita ser seguido.

\section{REFERÊNCIAS}

BIBIANO, B. Entrevista: Ignacio Sánchez. As razões do sucesso da universidade que ultrapassou a USP. Veja, Sao Paulo, Julho 2014. Disponible en: < http://veja.abril.com.br/ noticia/educacao/as-razoes-do-sucesso-da-universidade-que-ultrapassou-a-usp $>$. Acceso en: 19 julio 2014.

COMISIÓN ESPECIAL INVESTIGADORA SOBRE EL FUNCIONAMIENTO DE LA EDUCACIÓN SUPERIOR. Informe de la Comisión Investigadora Sobre el Funcionamiento de la Educación Superior. Santiago: Cámara de Diputados de Chile, 2012. 368 p. Disponible en: <http://www.cooperativa.cl/noticias/site/artic/20120624/asocfile/20120624130026/ pdf_1.pdf $>$. Acceso en: 19 julio 2014.

COMISIÓN ESPECIAL INVESTIGADORA SOBRE EL FUNCIONAMIENTO DE LA EDUCACIÓN SUPERIOR. Informe de la Comisión Especial Investigadora Encargada de Estudiar a Fondo el Sistema de Educación Superior Chilena. Santiago: Cámara de Diputados de Chile, 2014. 428 p. Disponible en: <http://educacion2020.cl/sites/default/files/ comision_especial_investigadora_del_sistema_de_la_educacion_superior_chilena_.pdf $>$. Acceso en: 19 julio 2014.

COMISIÓN NACIONAL DE ACREDITACIÓN. Aprueba Reglamento Sobre Áreas de Acreditación Acorde al Artículo $17^{\circ}$ de la Ley 20.129. Disponible en: < http:/ / www.cnachile. cl/Documentos\%20de\%20Paginas/RES-DJ-01.pdf>. Acceso en: 19 julio 2014.

CONTRALORIA GENERAL DE LA REPÚBLICA. Financiamiento Fiscal a la Educación Superior 2012. Santiago: División de Análisis Contable, 2013. 88 p. Disponible en: < http:// www.contraloria.cl/NewPortal2/portal2/ShowProperty/BEA\%20Repository/Portal/ Bases/Contabilidad/Estudios/Financiamiento_Fiscal_2012>. Acceso en: 19 julio 2014.

FRAGA, É; TAKAHASHI, F. Mensalidade na USP poderia ser paga por $60 \%$ dos alunos. Folha de São Paulo, São Paulo, Julho 2014. Disponible en: <http://www.folha.uol.com.br/ educacao/2014/06/1463535-mensalidade-na-usp-poderia-ser-paga-por-60-dos-alunos. shtml>. Acceso en: 19 julio 2014. 
FRONTAURA Y ARANA, J. M. Historia del Convictorio Carolino. (Apuntes para la historia de los antiguos colegios de Chile). Santiago: Imprenta Nacional, 1889. 82 p. Disponible en: <http:// www.memoriachilena.cl/archivos2/pdfs/MC0013869.pdf>. Acceso en: 19 julio 2014.

HUNEEUS, C. La Reforma Universitaria veinte años después. Santiago: Corporación de Promoción Universitaria, 1988. 117. Disponible en: <http://www.memoriachilena.cl/ archivos2/pdfs/MC0027769.pdf>. Acceso en: 19 julio 2014.

INSTITUTO NACIONAL DE EVALUACIÓN EDUCATIVA. Panorama de la Educación. Indicadores de la OCDE 2013. Informe Español. Madrid: Ministerio de Educación, Cultura y Deporte, 2013. 53 p.

MEDINA, J. T. Historia de la Real Universidad de San Felipe de Santiago de Chile. Santiago: Sociedad Imprenta y Litografía Universo, 1929. 650 p. Disponible en: <http://www.memoriachilena. cl/archivos2/pdfs/MC0001309.pdf>. Acceso en: 19 julio 2014.

MINISTERIO DE EDUCACIÓN PÚBLICA. Decreto Ley Nº 50, de 02 de Octubre de 1973. Designa Rectores Delegados en Universidades del país. Santiago. Disponible en: < http://bcn.cl/1m531>. Acceso en: 19 julio 2014.

MINISTERIO DE EDUCACIÓN PÚBLICA. Decreto Fuerza Ley N ${ }^{\circ} 1$, de 03 de Enero de 1981. Fija Normas sobre Universidades. Santiago. Disponible en: <http://bcn.cl/1m53t $>$. Acceso en: 19 julio 2014.

MINISTERIO DE EDUCACIÓN PÚBLICA. Ley N 18.433, de 04 de Septiembre de 1985. Crea la Universidad Metropolitana de Ciencias de la Educación. Santiago. Disponible en: <http://bcn.cl/1m5id>. Acceso en: 19 julio 2014.

MINISTERIO DE EDUCACIÓN PÚBLICA. Ley N 18.434 , de 04 de Septiembre de 1985. Crea la Universidad de Playa Ancha de Ciencias de la Educación. Santiago. Disponible en: <http://bcn.cl/1m5ii>. Acceso en: 19 julio 2014.

MINISTERIO DE EDUCACIÓN PÚBLICA. Decreto N 51, de 06 de Abril de 1999. Crea Comisión Asesora en materia de Evaluación de Calidad en Programas de Pregrado de Instituciones Autónomas de Educación Superior. Santiago. Disponible en: <http://bcn. cl/1m5ih>. Acceso en: 19 julio 2014.

MINISTERIO DE EDUCACIÓN. Ley N² 20.027, de 11 de Junio de 2005. Establece Normas para el Financiamiento de Estudios de Educación Superior. Santiago. Disponible en: <http://bcn.cl/1m5if>. Acceso en: 19 julio 2014.

MINISTERIO DE EDUCACIÓN. Ley Nº 20.129, de 17 de Noviembre de 2006. Establece un Sistema Nacional de Aseguramiento de la Calidad de la Educación Superior. Santiago. Disponible en: <http://bcn.cl/1m5di>. Acceso en: 19 julio 2014.

MINISTERIO DE EDUCACIÓN. Estados Financieros de las Universidades. Santiago: s/i, 2012. MINISTERIO DE EDUCACIÓN. Evolución de la Matrícula Total por Tipo de Institución (19832013). Santiago: s/i, 2013.

PROGRAMA INTERDISCIPLINARIO DE INVESTIGACIÓN EDUCATIVA. Las Transformaciones Educacionales bajo el Régimen Militar. Santiago: PIIE, 1984. 652 p.

SÁNCHEZ, P. Una asignatura pendiente. Profesores y profesoras detenidos/as desaparecidos/as y ejecutados/as en Cbile de 1973 a 1990. Santiago: Editorial ARCIS, 2013. 423 p.

UNIVERSIDAD DE SANTIAGO DE CHILE. Libro Memorial de la Universidad Técnica del Estado y de la Universidad de Santiago. Informe de la Comisión de Reconciliación Universitaria de 1991, actualizado a 40 años del golpe de Estado de 1973. Santiago: Sello Editorial USACH, 2013. 241 p. VÁSQUEZ, H. Una luz sobre la sombra. Detenidos desaparecidos y asesinados de la Pontificia Universidad Católica de Chile. Santiago: s/i, 2010. 180 p. 


\section{NOTAS}

${ }^{1}$ En el periódico se señalaba que cerca del $60 \%$ de los Estudiantes de la USP podrían pagar un arancel y que eso significaría recaudar cerca de 1,8 billones de reales anuales, entre otras cosas.

${ }^{2}$ Ver: http://www.topuniversities.com

${ }^{3}$ Ver: FRONTAURA Y ARANA, J. M. Historia del Convictorio Carolino. (Apuntes para la historia de los antiguos colegios de Chile). Santiago: Imprenta Nacional, 1889. 82 p. Disponible en: <http://www.memoriachilena.cl/archivos2/pdfs/MC0013869.pdf>. Acceso en: 19 julio 2014.

${ }^{4}$ Ver: MEDINA, J. T. Historia de la Real Universidad de San Felipe de Santiago de Chile. Santiago: Sociedad Imprenta y Litografía Universo, 1929. 650 p. Disponible en: <http:// www.memoriachilena.cl/archivos2/pdfs/MC0001309.pdf>. Acceso en: 19 julio 2014.

${ }^{5}$ La Universidad Técnica Del Estado asumió este nombre en el año 1947 y es la continuadora de la Escuela de Artes y Oficios (1849-1947) y otras Escuelas Politécnicas existentes en la época en Santiago y otras regiones. A partir de 1981 la Dictadura Militar la interviene y le asigna el nombre que mantiene hasta el día de hoy: Universidad de Santiago de Chile.

${ }^{6}$ En 1990 pasa a llamarse Universidad Católica del Norte.

${ }^{7}$ Un libro interesante para conocer sobre el proceso de Reforma Universitaria en Chile es el siguiente: HUNEEUS, C. La Reforma Universitaria veinte años después. Santiago: Corporación de Promoción Universitaria, 1988. 117 p. Disponible en: <http://www. memoriachilena.cl/archivos2/pdfs/MC0027769.pdf>. Acceso en: 19 julio 2014.

${ }^{8}$ Algunos libros que abordan la represión política en instituciones educacionales chilenas durante la Dictadura Militar son los siguientes: SÁNCHEZ, P. Una asignatura pendiente. Profesores y profesoras detenidos/as desaparecidos/as y ejecutados/as en Chile de 1973 a 1990. Santiago: Editorial ARCIS, 2013. 423 p. UNIVERSIDAD DE SANTIAGO DE CHILE. Libro Memorial de la Universidad Técnica del Estado y de la Universidad de Santiago. Informe de la Comisión de Reconciliación Universitaria de 1991, actualizado a 40 años del golpe de Estado de 1973. Santiago: Sello Editorial USACH, 2013. 241 p. VÁSQUEZ, H. Una luz sobre la sombra. Detenidos desaparecidos y asesinados de la Pontificia Universidad Católica de Chile. Santiago: s/i, 2010. 180 p.

${ }^{9}$ Abogado, Arquitecto, Bioquímico, Cirujano Dentista, Ingeniero Agrónomo, Ingeniero Civil, Ingeniero Comercial, Ingeniero Forestal, Médico Cirujano, Médico Veterinario, Psicólogo, Químico Farmacéutico. Es interesante observar como en esta demarcación de carreras exclusivamente universitarias fueron excluidos los campos de conocimiento de las Humanidades y de las Ciencias Puras. Un caso aún más complejo es el de las Pedagogías, puesto que en 1973 fueron cerradas las históricas Escuelas Normales donde se formaban docentes desde mediados del siglo XIX, quedando así reducida la formación de docentes solamente a las Universidades. En 1981 la formación docente también fue excluida dentro de este listado de carreras exclusivas de las Universidades, mostrando así el desinterés de las autoridades de la época con la pedagogía.

${ }^{10}$ Hasta 1981 funcionó el Instituto de Ciencias Pedagógicas de Valparaíso que fue desvinculado de la Universidad de Chile y pasó a llamarse Instituto Profesional Academia Superior de Ciencias Pedagógicas de Valparaíso. En el año 1985 pasa a conformarse como Universidad de Playa Ancha de Ciencias de la Educación. Ley 18.434.

${ }^{11}$ Hasta 1981 funcionó el Instituto Pedagógico de Santiago que fue desvinculado de la Universidad de Chile y pasó a llamarse Instituto Profesional Academia Superior de Ciencias Pedagógicas de Santiago. En el año 1985 pasa a conformarse como Universidad Metropolitana de Ciencias de la Educación. Ley 18.433. 
${ }^{12}$ La Trasnacional Laureate International Universities controla en Chile a las siguientes instituciones educacionales: Universidad Andrés Bello; Universidad de las Américas; Universidad Viña del Mar; Instituto Profesional AIEP; Escuela Moderna de Música. Es interesante resaltar que dos de estas Universidades son las que tienen en Chile los números mayores de estudiantes matriculados para el año 2013: Universidad Andrés Bello: 42.012 estudiantes; Universidad de las Américas: 34.362 estudiantes. Fuente: www.mifuturo.cl. Esta Trasnacional del área educacional es propietaria de instituciones educacionales en América Latina, Estados Unidos, Asía, Oceanía, Europa, Norte de África.

${ }^{13}$ A la Matrícula de Universidades del CRUCH de los años 1983, 1984 y 1985 se agregó la matrícula correspondiente a las Academias Pedagógicas que posteriormente pasaron a convertirse en Universidades propiamente tal.

${ }^{14}$ Ver: CONTRALORIA GENERAL DE LA REPÚBLICA. Financiamiento Fiscal a la Educación Superior 2012. Santiago: División de Análisis Contable, 2013. 88 p. Disponible en: < http://www.contraloria.cl/NewPortal2/portal2/ShowProperty/BEA\%20Repository/Portal/ Bases/Contabilidad/Estudios/Financiamiento_Fiscal_2012>. Acceso en: 19 julio 2014.

${ }^{15}$ Este aporte lo reciben además de las Universidades los Institutos Profesionales y los Centros de Formación Técnica.

${ }^{16}$ Ver: INSTITUTO NACIONAL DE EVALUACIÓN EDUCATIVA. Panorama de la Educación. Indicadores de la OCDE 2013. Informe Español. Madrid: Ministerio de Educación, Cultura y Deporte, 2013. 53 p.

${ }^{17}$ Con anterioridad a esta modalidad de financiamiento a través de créditos también existían otras alternativas similares, como el Fondo Solidario de Crédito Universitario y el Crédito CORFO, no obstante, el primero es delimitado para los estudiantes de las Universidades del CRUCH y ambos no son masivos.

${ }^{18}$ Sólo es obligatorio acreditar las Carreras vinculadas a las áreas de Salud y Educación.

${ }^{19} \mathrm{El}$ proceso y los alcances de la Acreditación son bastante elaborados y complejos. Aquí no se busca describir o explicar en su totalidad a la misma. Para saber más detalles se recomienda revisar la página web de la Comisión Nacional de Acreditación: www.cnachile.cl

${ }^{20}$ Ver: COMISIÓN NACIONAL DE ACREDITACIÓN. Aprueba Reglamento Sobre Áreas de Acreditación Acorde al Artículo $17^{\circ}$ de la Ley 20.129. Disponible en: <http:/ /www. cnachile.cl/Documentos\%20de\%20Paginas/RES-DJ-01.pdf>. Acceso en: 19 julio 2014.

${ }^{21}$ Universidad de las Américas; Universidad de Artes, Ciencias y Comunicación (UNIACC); Universidad La República; Universidad Bolivariana; Universidad Los Leones; Universidad Miguel de Cervantes; Universidad La Araucana; UCINF; Universidad de Aconcagua; Universidad Chileno-Británica de Cultura; Universidad del Mar; Universidad Pedro de Valdivia; Universidad Iberoamericana de Ciencias y Tecnología (UNICIT); Universidad SEK; Universidad Gabriela Mistral. Dentro de estas, la Universidad del Mar perdió su reconocimiento oficial debido a una serie de irregularidades arrastradas durante años.

${ }^{22}$ El día 06/07/2011, en pleno apogeo de las movilizaciones estudiantiles, se creó en la Cámara de Diputados de Chile la Comisión Especial Investigadora sobre el Funcionamiento de la Educación Superior, denominada popularmente como "Comisión Lucro". Dicha Comisión Investigadora ha presentado resultados interesantes de su trabajo: Ver: COMISIÓN ESPECIAL INVESTIGADORA SOBRE EL FUNCIONAMIENTO DE LA EDUCACIÓN SUPERIOR. Informe de la Comisión Investigadora Sobre el Funcionamiento de la Educación Superior. Santiago: Cámara de Diputados de Chile, 2012. 368 p. Disponible en: <http://www.cooperativa.cl/noticias/site/artic/20120624/asocfile/20120624130026/ 
pdf_1.pdf >. Acceso en: 19 julio 2014. COMISIÓN ESPECIAL INVESTIGADORA SOBRE EL FUNCIONAMIENTO DE LA EDUCACIÓN SUPERIOR. Informe de la Comisión Especial Investigadora Encargada de Estudiar a Fondo el Sistema de Educación Superior Chilena. Santiago: Cámara de Diputados de Chile, 2014. 428 p. Disponible en: $<$ http://educacion2020.cl/sites/default/files/comision_especial_investigadora_del_ sistema_de_la_educacion_superior_chilena_pdf>. Acceso en: 19 julio 2014.

Recebido: 22/07/2014

Aprovado: 02/12/2014

Contato:

Universidade Federal de Minas Gerais

$\mathrm{FaE}$ - GEPHE

Av. Pres. Antônio Carlos, 6627 - Pampulha

Belo Horizonte $|\mathrm{MG}|$ Brasil

CEP 31.270-901 
